SupportingInformation

\title{
Human VE-cadherin fusion protein as an artificial extracellular matrix enhancing the proliferation and differentiation functions of endothelial cell
}

\author{
Ke Xu ${ }^{l}$, Qizhi Shuai ${ }^{l}$, Xiaoning $\mathrm{Li}^{l}$, Yan Zhang ${ }^{l}$, Chao Gao ${ }^{l}$, Lei Cao ${ }^{1}$, Feifei Hu ${ }^{l}$,Toshihiro \\ Akaike $^{2}$, Jian-xi Wang ${ }^{3}$, Zhongwei Gu ${ }^{3}$, Jun Yang ${ }^{\text {I\# }}$
}

(1The Key Laboratory of Bioactive Materials, Ministry of Education, College of Life Science, Nankai University, Tianjin, 300071, China;

2Biomaterials Center for Regenerative Medical Engineering, Foundation for Advancement of International Science, Tsukuba, Japan;

3National Engineering Research Center for Biomaterials, Sichuan University, Chengdu, 610064, China;)

\#Corresponding author.

Prof. Jun Yang, The Key Laboratory of Bioactive Materials, Ministry of Education, College of life science, Nankai University, Tianjin, 300071, China

94 Weijin Road, Nankai District, Tianjin, China

E-mail address: yangjun106@nankai.edu.cn

Tel\& Fax: +0086 2223498038 

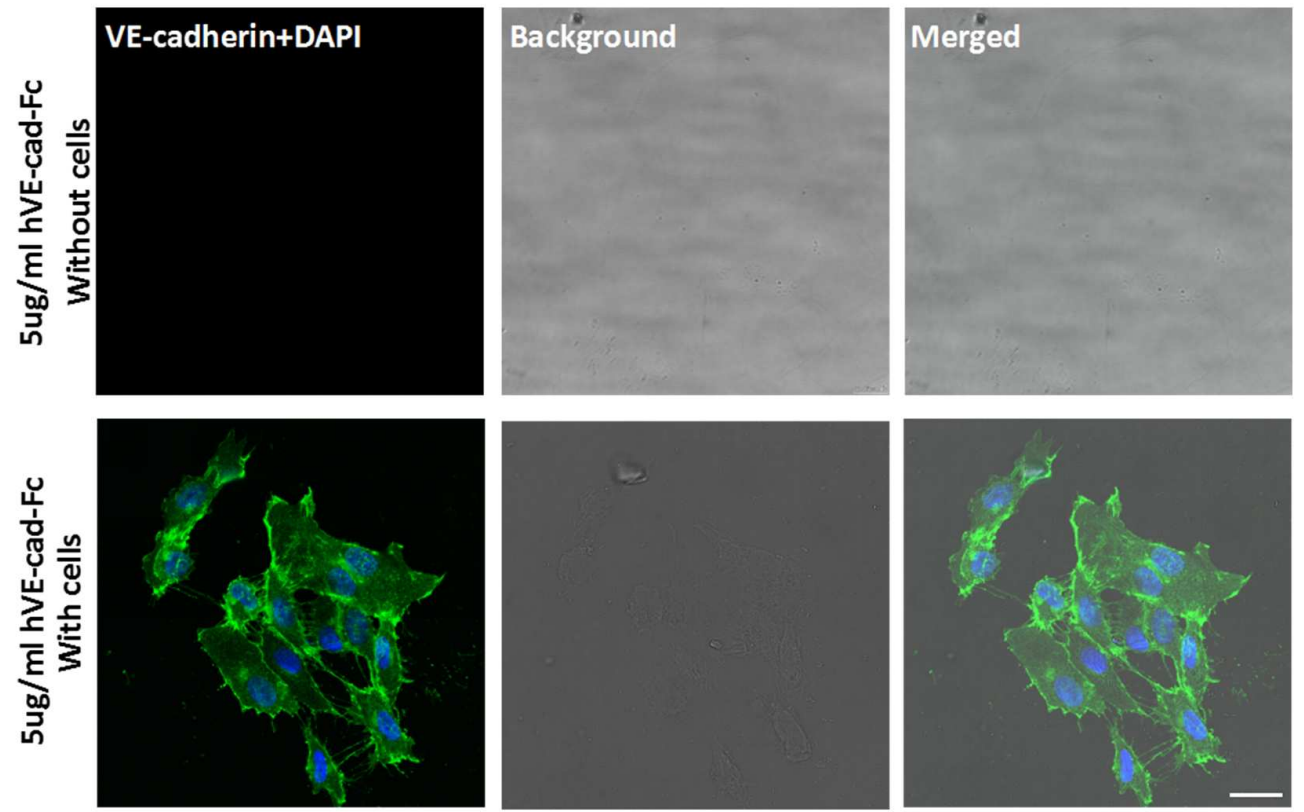

Figure S1. The immunofluorescence detection of recombinant fusion protein VE-cad-Fc and endogenous VE-cadherin on the hVE-cad-Fc matrix. The immunofluorescence staining of VE-cadherin on hVE-cad-Fc matrix with or without cells was observed at $24 \mathrm{~h}$ using confocal laser scanning microscopy. The results showed that the fusion protein could not be detected on the surfaces of plates by the immunostaining. Scale bars indicate $25 \mu \mathrm{m}$. All the results are representative of 3 independent experiments. 


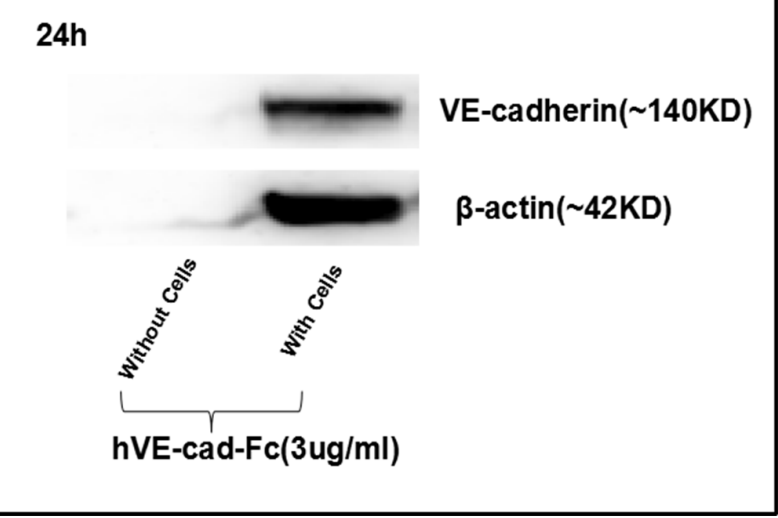

Fig S2. The western blotting analysis of recombinant fusion protein VE-cad-Fc and endogenous VE-cadherin on the hVE-cad-Fc matrix. VE-cadherin on hVE-cad-Fc matrix with or without cells was analyzed at $24 \mathrm{~h}$ using western blotting. The results showed that no band could be detected on the hVE-cad-Fc matrix without cells, implying that the fusion protein could not be separated by the cell lysis buffer from the PS plates. All the results are representative of 3 independent experiments. 